Paideusis

\title{
Le rôle de la philosophie de l'éducation dans la formation des maîtres
}

\section{Christiane Gohier}

Volume 4, Number 1, 1990

URI: https://id.erudit.org/iderudit/1073389ar

DOI: https://doi.org/10.7202/1073389ar

See table of contents

Publisher(s)

Canadian Philosophy of Education Society

ISSN

0838-4517 (print)

1916-0348 (digital)

Explore this journal

Cite this article

Gohier, C. (1990). Le rôle de la philosophie de l'éducation dans la formation des maîtres. Paideusis, 4(1), 9-13. https://doi.org/10.7202/1073389ar
Article abstract

We cannot talk about the role of philosophy of education in teachers' training programs without examining the relationship between philosophy and educational theories.

Even when theoreticians like Kieran Egan, for example, claim that education is a normative discourse that cannot be inferred from "local" empirical psychological research, the role of philosophy seems of utmost importance because philosophy has essentially to do with norms and values.

Another way of looking at the "sciences" of education that renews our comprehension of their relationship is by analysing a new cognitive psychological approach named "metacognition" which rests on one's ability to understand one's own cognitive strategies and mechanisms of regulation. The well-known psychologist Adrien Pinard in Quebec has been working on those concepts lately, inspired by Flavell's model of cognitive regulation. This approach is remarkably similar both to Wilson's definition of procedural criteria which he claims educational theory is all about and to the very specific method of philosophy, that of analytical and reflective thought. Philosophy or reflective thought cannot be separated from practice in teacher training since education is not only a matter of pedagogical means but also of attitudes to and relationship with another person. Thus, besides theory and practice, a third parameter should be taken into account in the elaboration of a teacher training program: the meta-practice and theory parameter, or the reflection on one's own practice, which is essentially philosophical. (c) Christiane Gohier, 1990

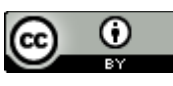

This document is protected by copyright law. Use of the services of Érudit (including reproduction) is subject to its terms and conditions, which can be viewed online.

https://apropos.erudit.org/en/users/policy-on-use/ 


\section{Le rôle de la philosophie de l'éducation dans la formation des maîtres.}

\section{Christiane Gohier Université du Québec à Montréal}

On ne peut aborder la question du rôle de la philosophie de l'éducation dans la formation des maîtres sans parler d'abord de l'apport ou du rapport de la philosophie à la théorie éducative.

Les points de vue, nous le savons, divergent sur cette question. La désignation institutionnelle du champ d'étude constitué par les questions éducatives illustre bien cette divergence. S'agit-il de l'éducation ou des sciences de l'éducation? Oeuvrons-nous au sein d'une faculté d'éducation ou d'une faculté des sciences de l'éducation? Ces dénominations sont la manifestation de deux positions antagonistes quant à la composition disciplinaire du discours éducatif.

Mises à part les discussions sur le statut scientifique de l'éducation, une autre question concernant son statut épistémologique revient en effet sans cesse, celle de sa spécificité. L'éducation est-elle une science à part entière ou est-elle d'emblée pluri ou interdisciplinaire, s'alimentant à des disciplines fondationnelles comme la philosophie, la psychologie, et la sociologie? La première tendance est représentée entre autres par le discours de théoriciens qui, comme Kieran Egan, considèrent que la théorie éducative devrait constituer un discours organisé en fonction de sa spécificité et ne recourir aux autres disciplines, comme la psychologie, que ponctuellement, lorsque celle-ci peut venir étayer le discours des éducateurs. Le principal argument invoqué est que du descriptif on ne peut déduire le normatif, encore moins d'un descriptif "local" issu d'une expérimentation très circonstanciée. (Du "is" on ne peut déduire le "ought").

Dans Education and Psychology, Egan dit en effet que pour avoir une implication pour la pratique éducative, les faits doivent être organisés dans le cadre d'une théorie éducative. ${ }^{1}$ Cette théorie éducative doit pouvoir prescrire un programme éducatif qui n'ait pas comme seul objectif de développer les habiletés de penser, mais également de former une personne. Egan décrit la bonne théorie éducative comme étant celle qui incluerait les éléments suivants:

The function of an educational theory is to tell us how to design a curriculum which will produce educated people, or rather, to lay out such a curriculum. The theory should tell us how we should teach such content. The theory should also tell us when we should teach particular things and how we should teach them at the different stages of educational development. It must tell us also what kind of person will be produced if we follow its prescriptions about what, how and when. This image of the end-product must justify the detailed prescriptions, making the latter causally responsible for the former. The theory will be culture-bound and value-saturated, and will involve claims about desirable political and social structures. ${ }^{2}$

Si Egan met en doute, jusqu'à un certain point, la contribution de la psychologie, il serait par ailleurs difficile avec une telle définition de mettre en doute celle de la philosophie, puisqu'il est question d'un discours normatif et prescriptif qui implique une conception de l'homme, de la sociétć et des valcurs, tous objets d'étude de la philosophie. On ne peut donc mettre en question 
l'apport de la philosophie à la théorie éducative, même dans une conception que l'on pourrait qualifier "d'autonomiste" du discours éducatif. Tous ne sont par ailleurs pas d'accord avec cette conception disciplinaire de l'éducation et optent plutôt pour une conception pluri et interdisciplinaire impliquant interrelation et apports mutuels de la psychologie et de la philosophie. Jonas Soltis et John Wilson entre autres défendent cette conception que j'ai désignée comme la conception de "sciences" (au pluriel) de l'éducation. Nous reviendrons plus loin sur les particularités de cette position.

$\mathrm{J}$ 'aimerais auparavant aborder la question des rapports entre psychologie et éducation d'une manière qui, je l'espère, nous aidera à renouveler la problématique psychologie-philosophie et éducation.

S'est en effet développé récemment un courant relativement nouveau en psychologie, celui de la métacognition, dont Monique Lefebvre-Pinard et Adrien Pinard sont les maitres d'oeuvre. S'inspirant entre autres des travaux de Flavell sur les modèles de régulation cognitive, ${ }^{3}$ ils ont élaboré un modèle de développement métacognitif, celui-ci étant défini comme le contrôle conscient par un individu de son propre fonctionnement cognitif. Ce contrôle requiert la possession par l'individu de deux composantes: le savoir métacognitif et les mécanismes d'autorégulation. Je me permettrai de faire état ici de la description que fait Pinard de ces deux composantes, car celle-ci est essentielle à la suite de mon propos sur les rapports de la philosophie et de l'éducation.

Pinard définit le savoir métacognitif comme

L'éventail des connaissances stables et verbalisables que l'individu a acquises 1) sur la diversité des objectifs possibles dans une démarche cognitive (p. ex.: one ne lit pas un texte de la même façon quand on le lit pour en faire la critique, pour en corriger la langue ou pour le réviser sur épreuves typographiques); 2) sur les différences inter- et intra-individuelles des personnes ( $y$ compris lui-même) engagées dans une telle démarche (p. ex: évaluer ses propres aptitudes dans une tâche donnée); 3) sur les différences entre diverses tâches cognitives en termes d'exigences (p. ex. une tâche en mathématiques, une tâche en litterature et une tâche en philosophie ont des contraintes très différentes); 4) sur les stratégies à utiliser pour progresser vers l'objectif visé de même que pour planifier, contrôler et évaluer l'efficacité de sa démarche (p. ex.: pour mieux assimiler un texte, il faut déjà connaître des moyens pour en dégager et en souligner les idées essentielles, en dépister les inconsistances, en faire une critique, etc. $)^{4}$

Quand au processus d'autorégulation, Pinard le définit essentiellement comme un processus de "feed-back" interne incluant les processus de planification d'ensemble, d'activation des stratégies nécessaires, de contrôle et vérification, etc. mis en ocuvre par un individu dans l'orchestration de ses connaissances au cours même d'une démarche cognitive. Ces processus reposent essentiellement sur ce que Flavell a appelé des expériences métacognitives, c'est-à-dire sur des pensées, impressions ou sentiments qui interrompent momentanément la démarche cognitive d'un individu aux prises avec un problème (p. ex. "il me faut redoubler de prudence parce que je ne suis pas très bon dans ce genre de problème"; "Ca ne va pas du tout, il faut changer de stratégie"...etc. $)^{5}$

Pinard identifie deux objectifs à la démarche métacognitive, soit l'optimisation de son propre développement intellectuel ou l'atteinte de ce que 
Vygotsky appelait la zone proximale de développement et la libération des contraintes imposées par le développement rapide de la technologie, soit le développement d'une pensée réflexive.

A la lecture et à la relecture tant des objectifs que des stratégies cognitives mises en jeu dans ce processus métacognitif, j'ai été fascinée par leur ressemblance évidente avec le mode de pensée qu'institue l'approche philosophique, c'est-à-dire la pensée analytique et réflexive, par le biais de la logique informelle et de la théorie de l'argumentation à l'oeuvre dans toute analyse, $\mathrm{y}$ inclus celle du corpus éducatif. Pensée réflexive, critique, introspection, développement optimal de soi, analyse de la pensée qui se pense elle-même, j'ai trouvé là l'essentiel de ce que la pensée philosophique met en oeuvre depuis plus de 2,000 ans. Serions-nous en train de boucler la boucle et de revenir au point de départ, au coeur du processus de la rationalité elle-même? Philosophie et psychologie se retrouvent ici avec pour objet commun l'éducation à la pensée elle-même.

C'est ce que d'une autre manière John Wilson et Barbara Cowell affirment lorsqu'ils disent que la théorie éducative ne doit pas être centrée sur la certitude ou la vérité absolue des contenus, mais plutôt sur les modes ou processus cognitifs ou "proceduraux" (procedural criteria) qui sont les suivants:

- willingness and ability to examine concepts analytically and judge the clarity of the discourse.

- looking carefully at our own emotional reactions to the discourse and to the background (often unconscious) reasons or causes of such reactions

- ability and willingness to attend seriously to others' points of view, to answer rather than to avoid questions, not to take arguments ad hominem or feel threatened by criticism.

- to be able to argue opposing viewpoints inside our heads rather than just warming uncritically to some general idea and in general to follow the Socratic model in our handling of such discourse. ${ }^{6}$

Ce discours rejoint celui de bien d'autres théoriciens en éducation qui réclament pour les maîtres une formation qu'ils appellent à juste titre fondamentale. En conclusion, force nous est de reconnaitre l'importance de la philosophie pour le discours éducatif.

Si l'on adopte le point de vue "autonomiste" sur l'éducation en revendiquant la spécificité de celle-ci comme discours normatif et prescriptif, mettant de l'avant une conception de l'homme, des valeurs, de la transmission du savoir, la philosophie comme discours réflexif ayant pour objet d'étude l'homme, aussi bien à travers la dimension éthique qu'axiologique, est interpellée. Si, au contraire, on valorise et favorise une conception pluridisciplinaire de l'éducation, en mettant plus particulièrement l'accent sur l'apport de la psychologie, on constate que les nouvelles tendances en psychologie cognitive vont dans le sens d'une métaréflexion, d'une auto-analyse du comportement cognitif qui se traduit par le courant de la métacognition. Or, la philosophie comme discours critique, incluant le discours sur l'argumentation, la logique, aussi bien que la logique informelle, est ici aussi interpellée au premier chef. 
Reconnaître l'importance de la philosophie pour le discours éducatif nous amène à reconnaître son importance pour la formation des maîtres qui est un des lieux privilégiés, le plus important sans doute, de son application. La problématique de la formation des maîtres est balisée par deux paramètres: la nécessité de la formation professionnelle, et celle de la formation théorique du futur enseignant. On pourrait mutatis mutandis appliquer à la problématique de la formation des maîtres ce que Jonas Soltis ${ }^{7}$ dit des paradigmes prévalents en éducation, soit l'approche normative ou philosophique de Dewey et l'approche empirique ou psychologique de Thorndike, à savoir qu'ils sont deux paradigmes complémentaires. De la même manière, on ne peut songer à une formation qui excluerait la pratique sur le terrain non plus qu'une réflexion par l'enseignant sur sa propre démarche intellectuelle ou sur sa pratique au niveau de l'intervention envers l'autre, autant au niveau des valeurs que de la démarche générale d'intervention. On n'a qu'a songer au cas exemplaire de l'éducation interculturelle qui met en cause non seulement la pratique pédagogique en tant que telle mais tout le rapport à l'autre. La philosophie, autant par sa méthode analytique et réflexive que par ses objets d'étude, l'homme et les valeurs, apparait ici, comme pour le discours éducatif lui-même, comme une discipline essentielle à la formation du maître dont on sait très bien qu'il n'est pas qu'informateur mais formateur d'homme, d'abord et avant tout à travers sa manière d'être et de penser.

La philosophie est en effet un discours auquel il faut avoir été formé pour se rendre compte qu'il est d'abord et avant tout discours de la méthode. De la dialectique platonicienne au doute méthodique cartésien, la philosophie, par un discours essentiellement analytique, forme l'esprit à la pensée réflexive dont l'objet peut varier à l'infini, puisque la pensée réflexive est la forme de la pensée elle-même.

C'est à ce titre que la philosophie est une discipline essentielle dans la constitution de ce qu'on désigne actuellement comme la formation "fondamentale", qu'au seizième siècle déjà Montaigne définissait comme la formation d'une "tête bien faite".

Si le discours philosophique comme méthode peut s'appliquer à tout objet de la pensée, comme champ théorique, il est par ailleurs balisé par des objets qui forment sa spécificité. La question de l'éthique ou de l'axiologie, soit des valeurs, par exemple, lui appartient en propre, ainsi que la question du rapport de l'homme-au-monde, faisant l'objet, entre autres, des théories de la connaissance.

Or, les théories ćducatives, comme nous venons de le voir, font appel à la philosophie dans sa double fonction: d'abord, puisqu'elles ont pour objet la formation de la personne, à l'aspect analytique du discours philosophique, puis, parce qu'elles sont prescriptives, à la réflexion d'ordre éthique et épistémologique.

Ainsi la réflexion philosophique participe-t-elle de deux des trois volets qui devraient constituer les fondements de tout programme de formation des maitres, soit la théorie, dont la réflexion philosophique est partie intégrante, la pratique, et la réflexion sur celles-ci que l'on pourrait qualifier de métatheorique et pratique, c'est-à-dire essentiellement philosophique. 


\section{Résumé}

We cannot talk about the role of philosophy of education in teachers' training programs without examining the relationship between philosophy and educational theories.

Even when theoreticians like Kieran Egan, for example, claim that education is a normative discourse that cannot be inferred from "local" empirical psychological research, the role of philosophy seems of utmost importance because philosophy has essentially to do with norms and values.

Another way of looking at the "sciences" of education that renews our comprehension of their relationship is by analysing a new cognitive psychological approach named "metacognition" which rests on one's ability to understand one's own cognitive strategies and mechanisms of regulation. The well-known psychologist Adrien Pinard in Quebec has been working on those concepts lately, inspired by Flavell's model of cognitive regulation. This approach is remarkably similar both to Wilson's definition of procedural criteria which he claims educational theory is all about and to the very specific method of philosophy, that of analytical and reflective thought. Philosophy or reflective thought cannot be separated from practice in teacher training since education is not only a matter of pedagogical means but also of attitudes to and relationship with another person. Thus, besides theory and practice, a third parameter should be taken into account in the elaboration of a teacher training program: the meta-practice and theory parameter, or the reflection on one's own practice, which is essentially philosophical.

\section{Notes}

' ${ }^{1}$ Kicran Egan, Education and Psychology, Plato, Piaget and Scientific Psychology, (New York and London: Teachers College Press, 1983), 109. (Traduction libre.)

${ }^{2}$ Ibidem, 124.

${ }^{3}$ Adrien Pinard, "Prise de conscience and taking charge of one's own cognitive functioning," Human Development, 29(3), 1987, 342.

${ }^{4}$ Adrien Pinard, "Cognition et métacognition: les recherches sur le développement de l'intelligence,"' Interface, 8(6), 1987, 20.

Ibidem, 20.

6John Wilson and Barbara Cowell, "Do We Need Theories in Education," Paideusis, 1(2), 1988, 2-11.

${ }^{7}$ Jonas Soltis, "Dewey and Thorndike: The Persistence of Paradigms in Educational Scholarship," Revue canadienne de l'éducation, 13(1), 1988, $39-51$. 